Article

\title{
A Full-Duplex LED-to-LED Visible Light Communication System
}

\author{
Hyunwoo Jung and Sung-Man Kim * \\ Department of Electronic Engineering, Kyungsung University, Nam-gu, Busan 48434, Korea; \\ hyunwoo125@hotmail.com \\ * Correspondence: sungman@ks.ac.kr
}

Received: 30 August 2020; Accepted: 14 October 2020; Published: 18 October 2020

check for updates

\begin{abstract}
We experimentally demonstrated full-duplex light-emitting diode (LED)-to-LED visible light communication (VLC) using LEDs as the transmitter and receiver. Firstly, we investigated the performance dependency on the wavelengths of the LED transmitter and receiver by measuring the rise time and signal-to-noise ratio (SNR). Through the investigation, we were able to choose the optimal LED color set for LED-to-LED VLC using Shannon's channel capacity law. The bit error rate (BER) results of full-duplex and half-duplex LED-to-LED VLC systems with the optimal LED sets are shown to compare the performance. Furthermore, we discuss major distortions and signal losses in the full-duplex LED-to-LED VLC system.
\end{abstract}

Keywords: visible light communication; LiFi; LED-to-LED; LED as a receiver

\section{Introduction}

Illumination trends have seen a move from incandescent bulbs and fluorescent lamps to LEDs due to their advantages over conventional illumination methods, such as their high power efficiency, long life time, and fast response time. Among these advantages of LEDs, their fast response characteristic makes their use possible not only for illumination, but also for wireless communication. Visible light communication (VLC), which is also called nm-wave communication, is a communication technology that uses visible light spectra from 380 to $740 \mathrm{~nm}$ as information carriers [1].

VLC has several advantages over conventional radio frequency (RF) wireless communication. Firstly, VLC has a huge unlicensed bandwidth of about $400 \mathrm{THz}$. Because most RF spectra below $10 \mathrm{GHz}$ have been already used for many purposes, wireless communication engineers are trying to use higher frequencies over $10 \mathrm{GHz}$. Thus, it is expected that VLC may be used in the near future because the VLC spectrum is an extension of the trend of using higher frequencies. Secondly, VLC can be easily realized using the existing LEDs already deployed in buildings for lighting purposes by simply adding a micro controller. As each LED can work as an access point (AP), VLC could be one of the key technologies for Internet of Things (IoT) or massive machine-type communication (mMTC), since multiple Aps and a large bandwidth are required for IoT to connect numerous devices with one another. Thirdly, VLC uses visible light as the carrier frequency, which does not cause electro-magnetic interference (EMI) with other electronic devices in contrast to the conventional RF communication. Fourthly, high-speed communication links over $10 \mathrm{~Gb} / \mathrm{s}$ can be easily set through the VLC link [2]. Thanks to its advantages, research studies on VLC have been widely conducted, including for the IEEE 802.15.7 VLC standard [3]. Comparative research of optical wireless communication (OWC) technologies is conducted in [4]. VLC based on space-division multiple access (SDMA) [5], software-defined radio (SDR) [6], a light-to-frequency converter [7], and an indoor positioning system (IPS) using VLC is studied in [8]. A full-duplex vehicular VLC system and cooperative VLC system with full-duplex relaying is studied in $[9,10]$. 
However, in reality, VLC has not been widely commercialized yet. We think that one of the main reasons is its insufficient commercialization strategies. In [11], IPS based on VLC was suggested as the first step of a VLC commercialization method, since IPS can achieve functionality without adding additional hardware in mobile devices. However, IPS based on VLC still requires additional optical receivers, such as photodiodes or image sensors. As another commercialization strategy of VLC, we suggest LED-to-LED VLC technology. LED-to-LED VLC is a VLC technique that uses LEDs as both the transmitter and receiver [12-15]. Because many devices such as smart phones or traffic lights already have built-in LED lamps, LED-to-LED VLC does not require additional optical devices for data transmission and reception in VLC links. Thus, it is expected that technologies such as IPS and mMTC could be easily realized through an LED-to-LED VLC scheme due to their low-complexity, low cost, and ease of implementation. We believe that LED-to-LED VLC will contribute to the wide spread of VLC.

To our knowledge, there have been only a few research studies on LED-to-LED VLC so far. In [12], a LED-to-LED VLC system using colored LEDs is proposed and a related networking protocol was shown. In [13], various usage cases of VLC are introduced, such as smartphone-to-LED, LED-to-smartphone, and LED-to-LED. In [14], a LED-to-LED VLC with software-based synchronization using microcontroller is introduced. In [15], the link performance of a half-duplex LED-to-LED VLC is shown by our research team. The frequency response of the receiver LED depending on the types of receiver circuits is also shown. However, the performance dependency on the wavelengths of a transmitter and a receiver LED has not been studied in detail in the previous studies.

Therefore, in this study, we investigate the performance dependency on the wavelengths of a transmitter and a receiver LED, by measuring the rise time and signal-to-noise ratio (SNR) with respect to the color of transmitter and receiver LEDs. Through the investigation, the optimal color set for a transmitter LED and a receiver LED is chosen. With the optimal LED color set, a full-duplex VLC is experimentally demonstrated with a direct-current-biased optical orthogonal frequency division multiplexing (DCO-OFDM) modulation scheme. Furthermore, we discuss distortions and signal losses in the full-duplex LED-to-LED VLC system.

\section{Positive Negative Junction}

A positive negative (PN) junction, a basic physical structure for a photodiode (PD) and a LED, is a combination of p-type and n-type semiconductors. LEDs are PN junction semiconductor devices that were originally designed to emit photons with energy smaller than or equal to the bandgap energy. In contrast, PDs are also PN junction semiconductor devices, which were originally made to generate electric currents by accepting photons with energy greater than or equal to the bandgap energy. In general, forward bias is applied to LEDs and reverse bias is applied to PDs according to their usage purposes. However, an LED can function as a photodetector when reverse bias is applied. The reason why LEDs can operate as optical emitters and optical receivers is because the physical structures of LEDs and PDs are the same. It was experimentally demonstrated that LEDs can be also utilized as optical receivers [15].

\section{Receiver Characteristics of LED}

In our experiment, we used HB10P red, yellow, green, blue, and white LEDs (Yinhui photoelectric). The size and viewing angle of the LEDs were $10 \varnothing(\mathrm{mm})$ and $30^{\circ}$, respectively. To obtain more detailed characteristics of the LEDs, we measured their spectra and optical powers. The measurement results are shown in Figure 1 and the parameters are summarized in Table 1 . The peak wavelengths for red, yellow, green, blue, and white LEDs were measured as 628, 587, 520, 465, and $454 \mathrm{~nm}$, respectively. Note that the white LED was a phosphor-converted white LED (pc-WLED). 


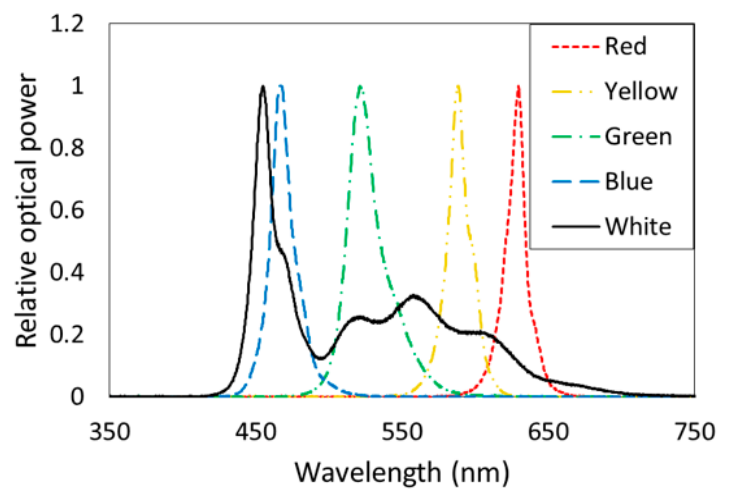

(a)

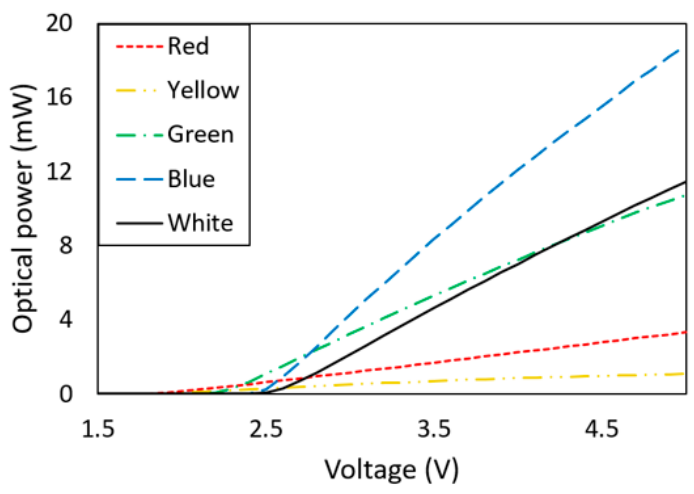

(b)

Figure 1. (a) The spectrum and (b) optical power of red, yellow, green, blue, and white light-emitting diodes (LEDs).

Table 1. Parameters of LEDs.

\begin{tabular}{cccccccc}
\hline \multicolumn{2}{c}{ Parameter } & Value & Dimension & \multicolumn{2}{c}{ Parameter } & Value & Dimension \\
\hline \multicolumn{2}{c}{ Viewing angle } & 30 & deg. & Diameter & 10 & $\mathrm{~mm}$ \\
\hline \multirow{6}{*}{ Peak } & Red & 628 & $\mathrm{~nm}$ & & Red & 1.168 & $\mathrm{~mW}$ \\
Wavelength & Yellow & 587 & $\mathrm{~nm}$ & Optical power & Yellow & 0.518 & $\mathrm{~mW}$ \\
& Green & 520 & $\mathrm{~nm}$ & (3V applied) & Green & 3.25 & $\mathrm{~mW}$ \\
& Blue & 465 & $\mathrm{~nm}$ & & Blue & 4.28 & $\mathrm{~mW}$ \\
& pc-white & 454 & $\mathrm{~nm}$ & & & & $\mathrm{~mW}$ \\
\hline
\end{tabular}

Shannon's channel capacity law is one of the powerful tools used to estimate the link performance when the channel bandwidth, average signal power, and average noise power are given. The channel capacity equation is given by:

$$
C=f_{3 d B} \cdot \log _{2}\left(1+\frac{E\left\{\overline{P_{s}}\right\}}{E\left\{\overline{P_{n}}\right\}}\right)
$$

where $C$ is the capacity of the channel, $f_{3 d B}$ is the $3 \mathrm{~dB}$ bandwidth of the channel, $P_{s}$ is the signal power, and $P_{n}$ is the noise power.

For a simple low-pass resistor capacitor $(\mathrm{RC})$ network, the step response of the system is given by:

$$
V(t)=V_{0}\left(1-e^{-\frac{t}{\tau}}\right)
$$

where $\tau$ is an RC time constant. The time that the signal takes to achieve $x \%$ of the voltage level can be obtained by solving Equation (2):

$$
t_{x \%}=-\tau \ln \left(1-\frac{x}{100}\right)
$$

Thus, the rise time $t_{r}$ can be expressed as:

$$
t_{r}=t_{0.9}-t_{0.1}=\tau \ln 9
$$

Since the $3 \mathrm{~dB}$ bandwidth of the low-pass RC system is given by

$$
f_{3 d B}=\frac{1}{2 \pi \tau}
$$

using Equations (4) and (5), the $3 \mathrm{~dB}$ bandwidth of a system can be approximated by the rise time of the received signal as:

$$
f_{3 d B}=\frac{\ln 9}{2 \pi t_{r}} \cong \frac{0.35}{t_{r}}
$$


Therefore, with Equations (1) and (6), we can derive another channel capacity equation:

$$
C \cong \frac{0.35}{t_{r}} \cdot \log _{2}\left(1+\frac{E\left\{\overline{P_{s}}\right\}}{E\left\{\overline{P_{n}}\right\}}\right)
$$

Using Equation (7), the approximated channel capacity of each LED color set (a transmitter and receiver LED set) can be calculated by the rise time, average signal power, and average noise power of the received signal.

Figure 2 shows the experimental setup for measuring the rise time and SNR for all of the possible combinations of color LED transmitters and color LED receivers. On the transmitter side, a square wave (SQW) is used to modulate the transmitter LED. A serial resistance of $15 \Omega$ is used in the transmitter circuit for circuit protection purposes. The frequency rate is $100 \mathrm{~Hz}$ and the transmission distance is $15 \mathrm{~cm}$. The optical signal is accepted through a receiver LED with a reverse bias of $5 \mathrm{~V}$ and a load resistance of $1 \mathrm{M} \Omega$. The electrical signal on the load resistance was measured by an oscilloscope (OSC). The rise time of the electrical signal is measured from $10 \%$ to $90 \%$ of the leading edge of the received signal.

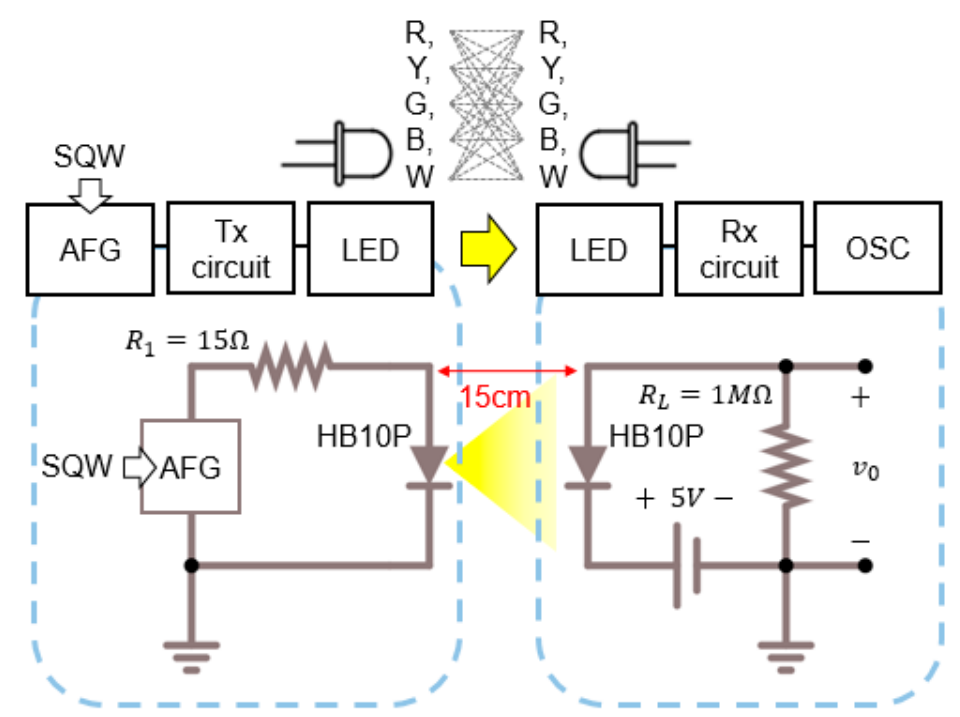

Figure 2. Experimental setup for measuring the rise time and signal-to-noise (SNR) of each LED color set (transmitter and receiver LEDs). Note that R, Y, G, B, and W stand for red, yellow, green, blue, and white, respectively.

Table 2 shows the experimental results of the rise time and SNR according to the pairs of transmitter and receiver LEDs. In addition, the channel capacities calculated by Equation (7) with the results for the rise time and SNR are shown in Table 3. Note that the three best performing cases in each experimental result are underlined in Tables 2 and 3. Additionally, the transmitter LED to pc-white LED case was not considered to avoid confusion, since pc-white LEDs are not appropriate receivers. In [15], a half-duplex LED-to-LED VLC performance study was conducted using a blue-to-green set, since the blue-to-green set showed the best received signal amplitude value, which is the same as our SNR results in Table 2.

However, the blue-to-green set is not the best case because its rise time is slow. The estimated channel capacity of blue-to-green set is $38.5 \mathrm{kbps}$, as shown in Table 3. According to Table 3, rather than the blue-to-green, instead the green-to-yellow set shows the best capacity of $59.1 \mathrm{kbps}$, which is $53 \%$ better than the blue-to-green set. Thus, the green-to-yellow set was chosen as the best color set for LED-to-LED VLC systems in our experiment. 
Table 2. Rise time and SNR of the LED-to-LED VLC according to the pairs transmitter and receiver LED color sets. Pc, phosphor-converted.

\begin{tabular}{|c|c|c|c|c|}
\hline \multirow[b]{2}{*}{ Transmitter } & \multicolumn{4}{|c|}{ Receiver } \\
\hline & $\begin{array}{c}\text { Red } \\
(628 \mathrm{~nm})\end{array}$ & $\begin{array}{l}\text { Yellow } \\
(587 \mathrm{~nm})\end{array}$ & $\begin{array}{l}\text { Green } \\
(520 \mathrm{~nm})\end{array}$ & $\begin{array}{c}\text { Blue } \\
(465 \mathrm{~nm})\end{array}$ \\
\hline $\operatorname{Red}(628 \mathrm{~nm})$ & $52 \mu \mathrm{s} / 2 \mathrm{~dB}$ & - & - & - \\
\hline Yellow (587 nm) & $80 \mu \mathrm{s} / 1 \mathrm{~dB}$ & $52 \mu \mathrm{s} / 6 \mathrm{~dB}$ & - & - \\
\hline Green (520 nm) & $72 \mu \mathrm{s} / 21 \mathrm{~dB}$ & $44 \mu \mathrm{s} / 22 \mathrm{~dB}$ & $96 \mu \mathrm{s} / 5 \mathrm{~dB}$ & - \\
\hline Blue (465 nm) & $63 \mu \mathrm{s} / 10 \mathrm{~dB}$ & $64 \mu \mathrm{s} / 11 \mathrm{~dB}$ & $112 \mu \mathrm{s} / \underline{37 \mathrm{~dB}}$ & $112 \mu \mathrm{s} / 12 \mathrm{~dB}$ \\
\hline Pc-white (454 nm) & $72 \mu \mathrm{s} / 11 \mathrm{~dB}$ & $64 \mu \mathrm{s} / 13 \mathrm{~dB}$ & $112 \mu \mathrm{s} / 25 \mathrm{~dB}$ & $120 \mu \mathrm{s} / 10 \mathrm{~dB}$ \\
\hline
\end{tabular}

The three best performing cases in each experimental result are underlined.

Table 3. Estimated channel capacity of the LED-to-LED VLC according to the pairs of transmitter and receiver LED color sets.

\begin{tabular}{ccccc}
\hline \multirow{2}{*}{ Transmitter } & \multicolumn{4}{c}{ Receiver } \\
\cline { 2 - 5 } & $\begin{array}{c}\text { Red } \\
\mathbf{( 6 2 8} \mathbf{~ n m})\end{array}$ & $\begin{array}{c}\text { Yellow } \\
\mathbf{( 5 8 7} \mathbf{~ n m})\end{array}$ & $\begin{array}{c}\text { Green } \\
\mathbf{( 5 2 0 ~} \mathbf{~ m})\end{array}$ & $\begin{array}{c}\text { Blue } \\
\mathbf{( 4 6 5} \mathbf{~ n m})\end{array}$ \\
\hline Red $(628 \mathrm{~nm})$ & $9.2 \mathrm{kbps}$ & - & - & - \\
\hline Yellow $(587 \mathrm{~nm})$ & $5.5 \mathrm{kbps}$ & $16.2 \mathrm{kbps}$ & - & - \\
\hline Green $(520 \mathrm{~nm})$ & $\underline{34 \mathrm{kbps}}$ & $\underline{59.1 \mathrm{kbps}}$ & $7.9 \mathrm{kbps}$ & - \\
\hline Blue $(465 \mathrm{~nm})$ & $19.4 \mathrm{kbps}$ & $20.2 \mathrm{kbps}$ & $\underline{38.5 \mathrm{kbps}}$ & $12.9 \mathrm{kbps}$ \\
\hline Pc-white $(454 \mathrm{~nm})$ & $18.4 \mathrm{kbps}$ & $24.9 \mathrm{kbps}$ & $25.6 \mathrm{kbps}$ & $10.4 \mathrm{kbps}$ \\
\hline & The three best performing cases are underlined. &
\end{tabular}

\section{Full-Duplex LED-to-LED VLC}

In previous studies, several modulation schemes have been studied for optical wireless communication. On-off keying (OOK) is the simplest and most conventional modulation method in optical communication, which has the advantages of low complexity and adequate bandwidth achievement. Pulse position modulation (PPM) is suggested in the IEEE 802.15.7 VLC standard for dimming support. PPM is another modulation scheme that can achieve higher power efficiency than OOK. However, PPM requires more complicated systems and bandwidth consumption [16]. Orthogonal frequency division multiplexing (OFDM) is a multi-carrier modulation scheme that shows high frequency efficiency. OFDM also makes systems robust to the low-frequency ambient light from other lighting sources by sending data via high-frequency subcarriers. In order to overcome the disadvantage of the low-data-rate and vulnerability to other lighting sources in LED-to-LED VLC, quadrature amplitude modulation (QAM) and DCO-OFDM are applied in our full-duplex LED-to-LED VLC experiment.

Figure 3 shows the experimental setup for the full-duplex LED-to-LED VLC link. A pseudo random binary sequence (PRBS) VLC signal is encoded with a 32-QAM and DCO-OFDM scheme. Hermitian symmetry input is used before the inverse fast Fourier transform (IFFT) process to generate a real and unipolar OFDM signal for an intensity modulation and direct detecting (IM/DD) system [17]. Then, the OFDM signal is directly modulated with an arbitrary function generator (AFG) and sent to a green transmitter LED. The VLC signal emitted by the green transmitter LED is detected by the yellow receiver LED. The received signal is recovered with a fast Fourier transform (FFT), inverse Hermitian symmetry, and 32-QAM de-mapping process. The transmitter and receiver circuits are shown in Figure 2. The DCO-OFDM modulated signal has an amplitude of $0.3 \mathrm{~V}$ and an offset of $2.9 \mathrm{~V}$. 
The single-sided bandwidth of a subcarrier $B$ in our full-duplex LED-to-LED VLC system can be calculated as:

$$
B=\frac{1}{2 T_{S}}
$$

where $T_{S}$ is the symbol time duration. The data rate of this system $D$ is:

$$
D=B\left(N_{f f t}-2\right) \log _{2} M
$$

where $N_{f f t}$ is the size of FFT and $M$ is the size of constellation. In our experiment, an FFT size of 100 and a constellation size of 32 are used. The experimental conditions are summarized in Table 4.

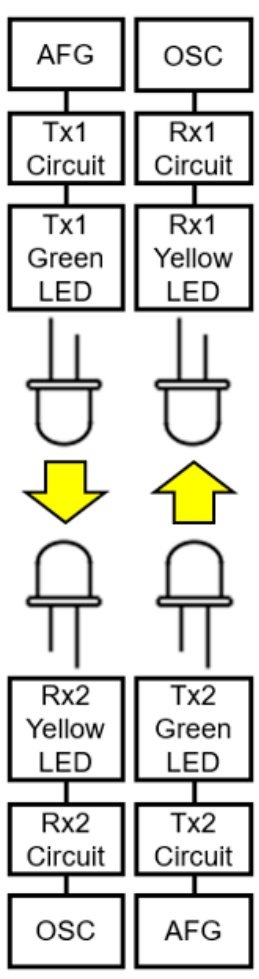

(a)

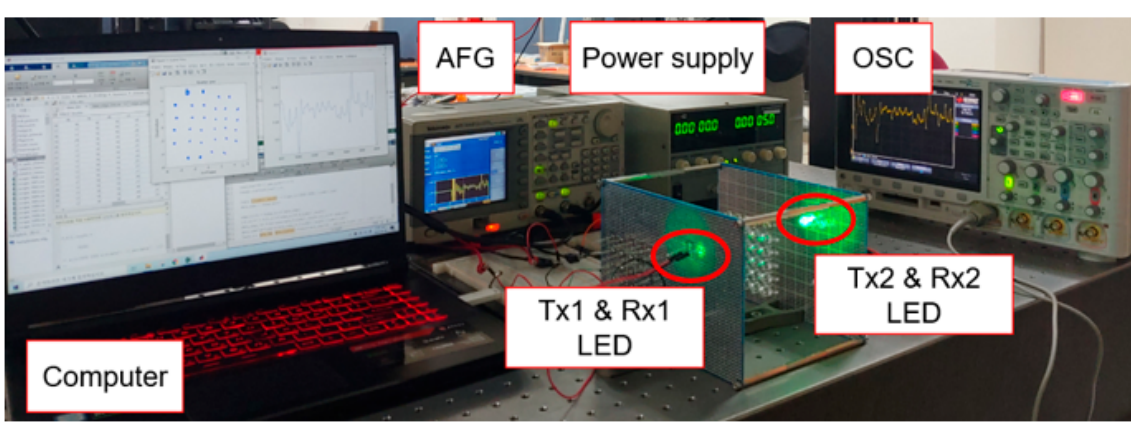

(b)

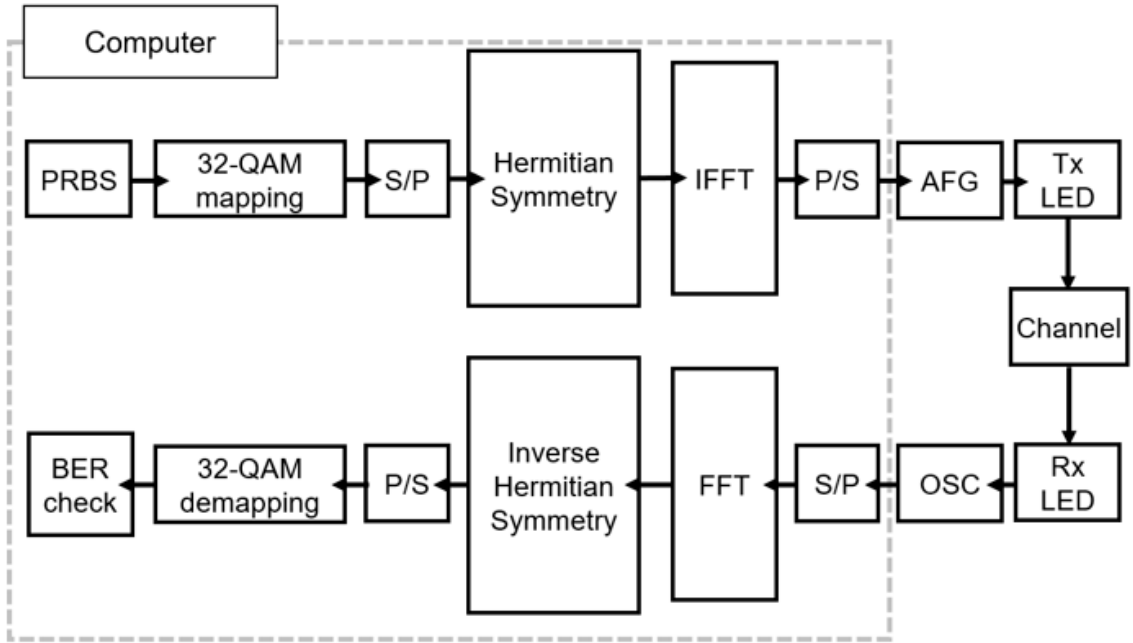

(c)

Figure 3. (a) Block diagram, (b) picture, and (c) detailed block diagram of the experimental setup for the full-duplex LED-to-LED VLC. Note that P/S and S/P stands for parallel-to-serial and serial-to-parallel, respectively.

Table 4. Experimental conditions in the full-duplex LED-to-LED VLC experiment.

\begin{tabular}{ccc}
\hline Parameter & Value & Dimension \\
\hline Modulation & DCO-OFDM & - \\
Tx offset & 2.9 & $\mathrm{~V}$ \\
Tx amplitude & 0.3 & $\mathrm{~V}$ \\
FFT size & 100 & - \\
QAM level & 32 & - \\
\hline
\end{tabular}

Figure $4 \mathrm{a}$ shows the interference path and signal path in the experimental setup, while Figure $4 \mathrm{~b}$ shows the bit error rate (BER) results of the half-duplex and the full-duplex LED-to-LED VLC. The received 32-QAM constellations are also shown in Figure 4b. At data rates of 4.9, 12.25, and 24.5 
kbps, the BER results are under $10^{-5}$ for both the half- and full-duplex systems. When the data rate is $49 \mathrm{kbps}$, the BERs of the half- and full-duplex systems are $6.25 \times 10^{-5}$ and $2.5 \times 10^{-4}$, respectively. When the data rate is $122.5 \mathrm{kbps}$, the BERs of the half- and full-duplex systems are 0.1604 and 0.1621 , respectively. Note that the reason why we only considered a few bit rates (i.e., 4.9, 12.25, 24.5, 49, and $122.5 \mathrm{kbps})$ in the experiment is that those bit rates showed the optimum sample rates in the experimental equipment.

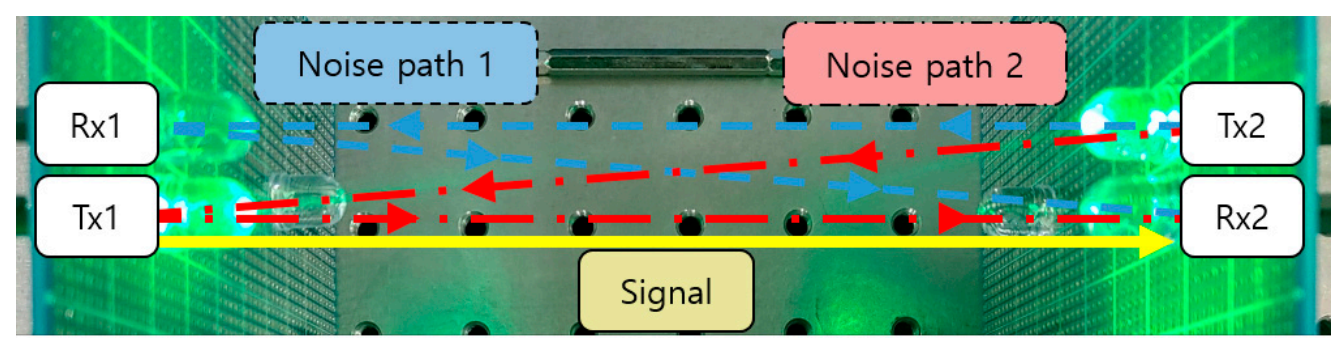

(a)

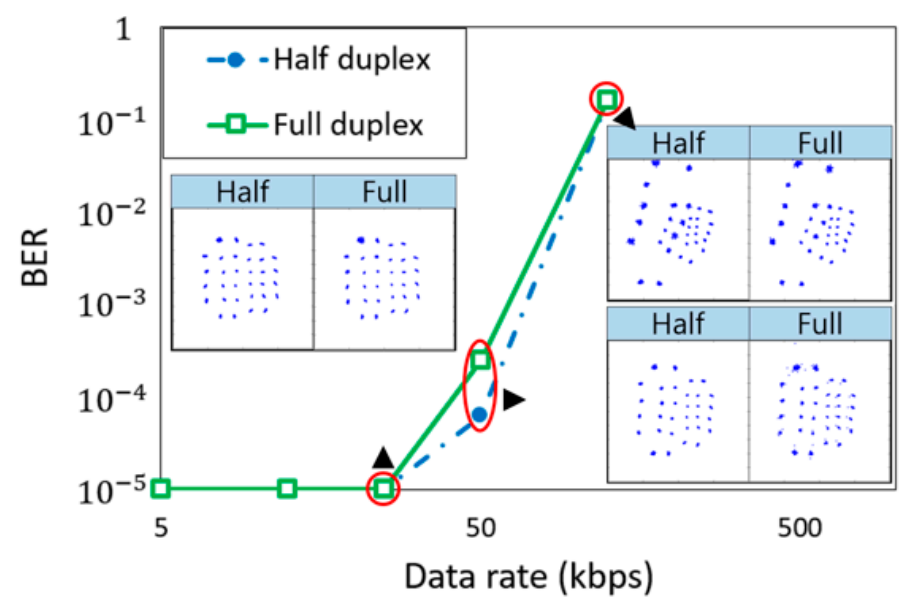

(b)

Figure 4. (a) Interference path (noise path 1 and noise path 2) and signal path in the experimental setup. (b) BER results and received constellations of the half-duplex and full-duplex LED-to-LED VLC systems.

These experimental results show that both half- and full-duplex LED-to-LED VLC systems with a data rate of $49 \mathrm{kbps}$ can be implemented, assuming the maximum allowable BER threshold is $10^{-3}$. Additionally, our system shows a channel capacity value similar to that of Shannon's channel capacity. The results also show that the performance of the half-duplex case is slightly better than the full-duplex case. We think that the reason for the performance difference is that the light emitted from the Tx2 LED is reflected at the surface of the Tx1 and Rx1 LED, then the Rx2 receives the reflected signal, as shown in Figure 4a. Based on this, we analyzed the interference effect mathematically.

The luminous intensity of a transmitter LED at an irradiance angle of $(\theta, \varnothing)$ is given by:

$$
I(\theta, \varnothing)=I(0,0) \cos ^{m}(\theta, \varnothing)
$$

where $I(0,0)$ is the center luminous intensity of the LED and $m$ is the Lambertian radiation pattern [18]. Analytic expression for the SNR of the half-duplex system is derived as:

$$
S N R_{H}=\frac{R_{p}\left(1-R_{f}\right) \int_{0}^{2 \pi} \int_{0}^{\tan ^{-1}\left(\frac{r}{d}\right)} I(\theta, \varnothing) d \theta d \varnothing}{P_{n}}
$$


where $R_{p}$ is the responsivity of the receiver LED, $d$ is the transmit distance, $r$ is the radius of the LED, $R_{f}$ is the reflectance of the fused silica (pure glass) at a wavelength of $589 \mathrm{~nm}$, and $P_{n}$ is the measured average ambient noise power in the receiver LED. The analytic expression for the SNR of the full-duplex system is derived as:

$$
S N R_{F}=\frac{R_{p}\left(1-R_{f}\right) \int_{0}^{2 \pi} \int_{0}^{\tan ^{-1}\left(\frac{r}{d}\right)} I(\theta, \varnothing) d \theta d \varnothing\left\{1+2 R_{f} \sin \left(2 \tan ^{-1} \frac{d_{t r}}{d}\right) \cos \left(\cos ^{-1} \frac{d_{t r}}{d}\right)\right\}}{P_{n}+2 R_{p} R_{f}\left(1-R_{f}\right) \int_{0}^{2 \pi} \int_{0}^{\tan ^{-1}\left(\frac{r}{d}\right)} I(\theta, \varnothing) d \theta d \varnothing \cos ^{2}\left(\cos ^{-1} \frac{d_{t r}}{d}\right)}
$$

where $d_{t r}$ is the distance between Rx 1 and Tx 1 LEDs and for Rx 2 and Tx2 LEDs. The values for each parameter are listed in Table 5. As shown in Figure 5, the analytic results and experimental results give similar curves. Thus, we can conclude that the interference mostly occurs due to the noise path shown in Figure 4a. As a result, it seems that slight performance degradation in the full-duplex system is caused by the reflection of the opposite LEDs.

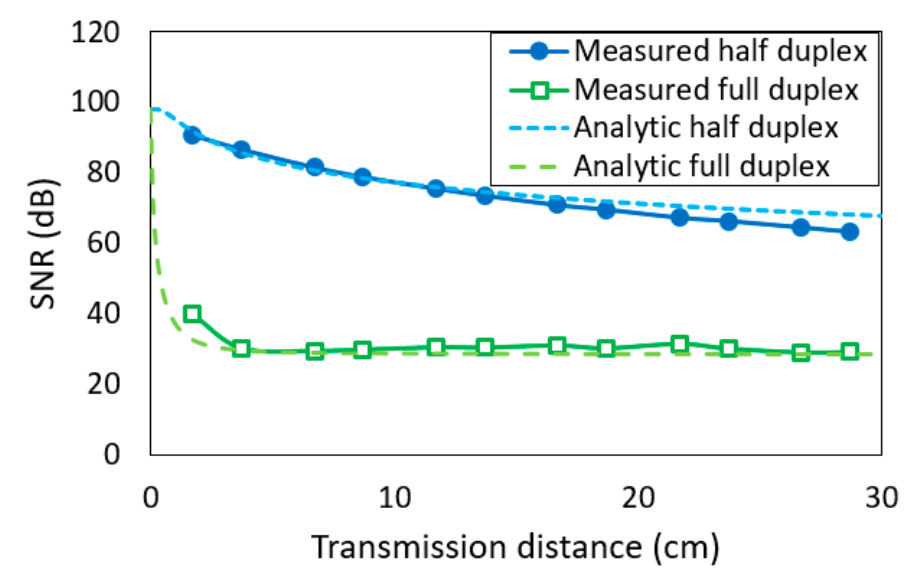

Figure 5. Analytical and measured SNR values for the half-duplex and full-duplex LED-to-LED VLC systems.

Table 5. Parameters used in the SNR analysis of half- and full-duplex LED-to-LED VLC systems.

\begin{tabular}{ccc}
\hline Parameter & Value & Dimension \\
\hline $\mathrm{I}(0)$ & $2.09 \times 10^{-3}$ & $\mathrm{~W} / \mathrm{sr}$ \\
$\mathrm{m}$ & 4.8188 & - \\
$R_{p}$ & $5.6 \times 10^{-4}$ & $\mathrm{~A} / \mathrm{W}$ \\
$r$ & 0.005 & $\mathrm{~m}$ \\
$R_{f}$ & 0.0186 & - \\
$P_{n}$ & $2.4 \times 10^{-21}$ & $\mathrm{~W}$ \\
$d_{t r}$ & 0.013 & $\mathrm{~m}$ \\
\hline
\end{tabular}

Since the transmission distance in our system was short, the optical signal power was much stronger than the ambient background light. Thus, the effect of ambient light from other lights can be ignored in our experimental results. Therefore, we think that the main reason for the signal degradation is the reflection of opposite LEDs.

As shown in our results, the LED-to-LED VLC system has a few limitations, such as its low data rate and short transmission distance. However, we think that a higher data rate and longer transmission distance can be achieved with advanced detection techniques (e.g., artificial neural-network-based detection and optimally weighted non-coherent detection) $[19,20]$.

In LED-to-PD VLC such as in [21], the data rate is much higher than for LED-to-LED VLC. The PD used in [21] (a New Focus 1601FS-AC instrument) had a rise time value of 400 ps and a peak responsivity value of $0.5 \mathrm{~A} / \mathrm{W}$. In contrast, our LED-to-LED VLC system achieved a maximum data rate 
of about $50 \mathrm{kbps}$, because the receiver LED had a rise time value of $44 \mu \mathrm{s}$ and a peak responsivity value of $0.56 \mathrm{~mA} / \mathrm{W}$. It is obvious that PDs shows much better performance than LEDs as optical receivers. However, we believe that LED-to-LED VLC can be commercialized because it has several advantages, such as its low complexity and low cost.

\section{Summary}

In summary, we investigated the performance dependency on the wavelength of the transmitter and the receiver LED for LED-to-LED VLC. In order to find the optimal color set of transmitter and receiver LEDs according to Shannon's channel capacity law, we measured the SNR and rise time of the LED-to-LED VLC system with all possible color combinations for the transmitter and receiver LEDs. Through the optimization process, we found out the optimal LED color set for LED-to-LED VLC, which was a green-to-yellow color set.

In addition, we experimentally demonstrated half- and full-duplex LED-to-LED VLC using a green LED and a yellow LED as the transmitter and the receiver, respectively. The maximum data rate of $49 \mathrm{kbps}$ with a BER of $<2.5 \times 10^{-4}$ was achieved using 32-QAM DCO-OFDM modulation and a reverse-biased circuit in a full-duplex LED-to-LED VLC system. We also investigated the interference effect in the full-duplex LED-to-LED VLC analytically and experimentally. We believe that LED-to-LED VLC technology will contribute to the wide spread of the VLC technology.

Author Contributions: Conceptualization, S.-M.K.; methodology, H.J.; software, H.J.; validation, H.J. and S.-M.K.; formal analysis, H.J.; investigation, H.J.; resources, H.J. and S.-M.K.; data curation, H.J.; writing-original draft preparation, H.J.; writing—review and editing, S.-M.K.; visualization, H.J.; supervision, S.-M.K.; project administration, S.-M.K.; funding acquisition, S.-M.K. All authors have read and agreed to the published version of the manuscript.

Funding: This research was supported by the National Research Foundation of Korea (NRF) grant funded by the Korea government (MSIT) (No. NRF-2019R1F1A1045956).

Conflicts of Interest: The authors declare no conflict of interest.

\section{References}

1. Haas, H.; Yin, L.; Wang, Y.; Cheng Chen, C. What is LiFi? J. Light. Technol. 2016, 34, 1533-1544. [CrossRef]

2. Bian, R.; Tavakkolnia, I.; Haas, H. 15.73 Gb/s Visible Light Communication with Off-the-Shelf LEDs. J. Light. Technol. 2019, 37, 2418-2424. [CrossRef]

3. Rajagopal, S.; Roberts, R.D.; Lim, S. IEEE 802.15.7 visible light communication: Modulation schemes and dimming support. IEEE Commun. Mag. 2012, 50, 72-82. [CrossRef]

4. Chowdhury, M.Z.; Hossan, M.T.; Islam, A.; Jang, Y.M. A Comparative Survey of Optical Wireless Technologies: Architectures and Applications. IEEE Access 2018, 6, 9819-9840. [CrossRef]

5. Kim, S.-M.; Lee, H.J. Visible light communication based on space-division multiple access optical beamforming. Chin. Opt. Lett. 2014, 12, 120601.

6. Martinek, R.; Danys, L.; Jaros, R. Visible Light Communication System Based on Software Defined Radio: Performance Study of Intelligent Transportation and Indoor Applications. Electronics 2019, 8, 433. [CrossRef]

7. Martínez Ciro, R.A.; López Giraldo, F.E.; Betancur Perez, A.F.; Luna Rivera, M. Characterization of Light-To-Frequency Converter for Visible Light Communication Systems. Electronics 2018, 7, 165. [CrossRef]

8. Tran, H.Q.; Ha, C. Fingerprint-Based Indoor Positioning System Using Visible Light Communication-A Novel Method for Multipath Reflections. Electronics 2019, 8, 63. [CrossRef]

9. Masini, B.M.; Bazzi, A.; Zanella, A. Vehicular visible light networks with full duplex communications. In Proceedings of the 5th IEEE International Conference on Models and Technologies for Intelligent Transportation Systems, Naples, Italy, 26-28 June 2017; pp. 98-103.

10. Narmanlioglu, O.; Kizilirmak, R.C.; Miramirkhani, F.; Uysal, M. Cooperative Visible Light Communications with Full-Duplex Relaying. IEEE Photonics J. 2017, 9, 1-11. [CrossRef]

11. Jovicic, A.; Li, J.; Richardson, T. Visible light communication: Opportunities, challenges and the path to market. IEEE Commun. Mag. 2013, 51, 26-32. [CrossRef] 
12. Giustiniano, D.; Tippenhauer, N.O.; Mangold, S. Low-complexity Visible Light Networking with LED-to-LED communication. In Proceedings of the IFIP Wireless Days, Dublin, Ireland, 21-23 November 2012; pp. 1-8.

13. Corbellini, G.; Aksit, K.; Schmid, S.; Mangold, S.; Gross, T.R. Connecting networks of toys and smartphones with visible light communication. IEEE Commun. Mag. 2014, 52, 72-78. [CrossRef]

14. Schmid, S.; Corbellini, G.; Mangold, S.; Gross, T.R. An LED-to-LED Visible Light Communication system with software-based synchronization. In Proceedings of the IEEE Globecom Workshops, Anaheim, CA, USA, 3-7 December 2012; pp. 1264-1268.

15. Kim, S.-M.; Lee, H.J. Half-duplex visible light communication using an LED as both a transmitter and a receiver. Int. J. Commun. Syst. 2016, 29, 1889-1895. [CrossRef]

16. Mahdiraji, G.A.; Zahedi, E. Comparison of Selected Digital Modulation Schemes (OOK, PPM and DPIM) for Wireless Optical Communications. In Proceedings of the 4th Student Conference on Research and Development, Selangor, Malaysia, 27-28 June 2006; pp. 5-10.

17. Armstrong, J. OFDM for Optical Communications. J. Light. Technol. 2009, 27, 189-204. [CrossRef]

18. Komine, T.; Nakagawa, M. Fundamental analysis for visible-light communication system using LED lights. IEEE Trans. Consum. Electron. 2004, 50, 100-107. [CrossRef]

19. Sönmez, M. Artificial neural network-based threshold detection for OOK-VLC Systems. Opt. Commun. 2020, 460, 125107. [CrossRef]

20. Hu, W.; Wei, Z.; Popov, S.; Leeson, M.; Zhang, M.; Xu, T. Non-Coherent Detection for Ultraviolet Communications with Inter-Symbol Interference. J. Light. Technol. 2020, 38, 4699-4707. [CrossRef]

21. Tsonev, D.; Chun, H.; Rajbhandari, S.; McKendry, J.D.; Videv, S.; Gu, E.; Haji, M.; Watson, S.; Kelly, A.E.; Faulkner, G.; et al. A 3-Gb/s Single-LED OFDM Wireless VLC Link Using a Gallium Nitride $\mu$ LED. IEEE Photonics Technol. Lett. 2014, 26, 637-640. [CrossRef]

Publisher's Note: MDPI stays neutral with regard to jurisdictional claims in published maps and institutional affiliations. 